\title{
Occurrence of cancer in Asians and non-Asians
}

\author{
L J DONALDSON AND D G CLAYTON \\ From the Department of Community Health, University of Leicester, Leicester Royal Infirmary, P O Box 65, \\ Leicester LE2 $7 L X, U K$
}

SUMMARY Cancer registration data for a defined geographical area, covering a seven year period, were modified to include the variable "Asian ethnic origin." The data were then used to test the hypothesis that a difference would be found between Asians and non-Asians in the pattern of cancer by site. Whereas the incidence of cancer of all sites appeared to be significantly lower in Asians $(\mathrm{p}<0.05)$, after taking account of this, and adjusting for the very different age distributions of the two populations, a highly significant difference $(p<0.0005)$ was found between the two groups in the distribution of cancer between sites. Although, given the size and young age structure of the Asian population, absolute numbers of cases were small, a significant excess of Asian cases (compared with the expected) occurred for cancer of the tongue, oral cavity, pharynx, and oesophagus. For most sites there were fewer Asian cases than would be expected, particularly so for the stomach, testis, and skin. The results indicate the need for formal epidemiological study to test specific aetiological hypotheses which may account for these apparent differences.

An earlier report used data that are systematically collected on hospital inpatients to compare the pattern of hospital morbidity in Asians and in non-Asians. ${ }^{1}$ There is a need to use other sources of health data to continue to explore apparent differences between the disease experience of various ethnic groups. Such studies could provide the basis for the formal testing of aetiological hypotheses and perhaps, ultimately, for planning preventive strategies.

Not only is cancer one of the leading causes of death in the indigenous population, it is now acknowledged that environmental factors must play a leading part in its causation. ${ }^{2}$ It seems, therefore, an appropriate group of diseases to study where there are populations that may differ considerably in dietary practices as well as other aspects of their lifestyles (and too in genetic predisposition).

All such studies are currently hampered by the fact that valid recording of ethnic origin is not a feature of most health records, nor is adequate demographic information available to allow the comparison of rates. $^{3}$

In this study cancer registration data have been modified to enable the pattern of cancer at different sites to be compared in Asians and non-Asians.

\section{Method}

The study population was the Leicestershire Health District (henceforth described as "Leicestershire"), a mixed urban and rural area containing a total resident population of about 840000 , of whom 55-60 000 are described as Asian. The meaning of "Asian origin" in this study is a person not of United Kingdom descent who originates from India, Pakistan, or Bangladesh or a person of Indian or Pakistani descent who originates from East Africa. In Leicestershire the Asian population (roughly 60\% Hindu, 25\% Muslim, and 15\% Sikh) came mainly from India (Gujerat and Punjab), Kenya, and Uganda. This health district has the same geographical boundaries as the Leicestershire Area Health Authority (Teaching) which it replaced at the time of the administrative restructuring of the National Health Service in April 1982.

A listing of all registrations of cancer occurring among Leicestershire residents over a seven year period (1976-82) was obtained from the Trent Regional Cancer Registry. From this total of 19254 registrations, 251 patients were identified with names of Asian appearance. This is considered to be a more reliable method of identifying a person of 
Asian ethnic origin than using country of birth. ${ }^{4}$ It has been used to study the pattern of Asian and non-Asian morbidity in hospitals. ${ }^{1}$ The possibility, through the use of names, of serious under enumeration of Asian women by this method due to cross cultural marriage is unlikely because this is estimated to be low in Britain now. ${ }^{5}$ Having been identified, the variable of Asian ethnic origin was added to the computer records of all relevant registrations. To preserve confidentiality, no names were included on the computerised record and data are presented in aggregated form.

Data on birthplace and parental birthplace derived from the 1971 census provided a crude estimate of the size and structure of the population of New Commonwealth ethnic origin around the time of that census. Below the national level, updated estimates in the succeeding years could not be produced reliably. ${ }^{6}$

Thus, for most of the period of the data being considered in the present study, no valid denominator existed to allow the cancer registration rate, by site, to be compared in Asians and non-Asians.

An alternative index of comparison was therefore the proportional registration ratio (PRR) adjusted for age to take account of the fact that the Asian population is generally much younger than the non-Asian population. The PRR was calcuiated by fitting the log linear model to the age group $\times$ site $\times$ ethnic group contingency table. The model fitted includes all first order associations and the PRRs are the parameters of the site $\times$ ethnic group term.

The 1981 census did not incorporate questions on parents' country of birth (included in the previous census). Data derived from it, however, which characterise those enumerated by the country of birth of their household head, provide a reasonable approximation of the size of the main ethnic minority populations in centres where their concentration is relatively high. ${ }^{6}$

Such data were helpful in the present study, since the study of proportional registrations by site cannot indicate whether the absolute incidence of cancer in Asians differed from that in non-Asians. The previous study of hospital morbidity suggested that it might be lower. ${ }^{1}$

Thus the 1981 census data for Leicestershire indicating the country of birth of the person's head of household were used to calculate an all site age standardised registration ratio (SRR) for Asians. This indirect standardisation was carried out in the usual way, cancer registrations in the three year period 1980-2 being taken. There were 130 Asian cases and 8268 non-Asian cases. The calculation yielded an SRR of $68 \%$ (95\% confidence limits,
57-81\%). The log linear model was parametised such that the product of the PRR and the SRR for all sites yields the site specific SRR. Thus the all site SRR for the three year data was multiplied by the PRR for each site in the seven year data and provided an estimated SRR for Asians, by site, for the whole study period.

\section{Results}

The table shows observed registrations of cancer by site and ethnic group, together with expected frequencies under the hypothesis of independence of site and ethnic group within age group. It also shows PRRs and estimates of site specific SRRs calculated as described above. The discrepancy between the observed and expected frequencies was assessed finally by a conventional chi squared test $(131.394$ on 43 degrees of freedom) which was highly significant $(\mathrm{p}<0.0005)$.

The table shows that the greatest number of Asiane cases compared with the expected occurred in the tongue, oral cavity, pharynx, and oesophagus. $\stackrel{\oplus}{\omega}$ Similarly, using the indication of an estimated SRR 0 N that was clearly greater than unity, other sites where $\stackrel{0}{0}$ Asian cases appeared to occur more frequently than $\stackrel{\mathbb{Q}}{\supset}$ expected were the thyroid gland, cervix, and other or $\mathbb{Q}$ unspecified malignancies of the lymph nodes. As is $\widetilde{D}$ also evident from the table, for most sites there were $\cap$ 맘 fewer Asian cases than would be expected given the $\frac{0}{3}$ pattern for non-Asians. This effect was particularly pronounced for some sites-for example, the skin, stomach, and testis.

These differences were further investigated by looking at the contribution of each of the 44 sites to the overall chi squared test. The interpretation of these contributions is difficult because many of the frequencies are small and there is also the problem of multiple comparisons. To avoid some of the difficulties we have used an approach based on Monte Carlo simulation and order statistics plots. Under the "null" hypothesis of independence between ethnic origin and site within age groups, we generated 99 computer simulated data sets showing the same marginal distributions of cancers among sites and among ethnic groups. For each such simulation we ranked the 44 separate contributions to the chi squared test for association. The figure plots the observed contribution ranked ith (from table 1) against the mean of the ith contribution in the 99 simulations. The plot also shows the $1 \%$ and $5 \%$ simulation envelopes. These are given by plotting against them the mean of the ith ranked contributions: $(a)$ the largest value achieved for the ith ranked contribution (1\% envelope) and $(b)$ the fifth largest achieved (5\% envelope). Since many 
"Observed" cancer registrations by site and ethnic group compared with "expected" frequencies. Shown also are two summary indices of comparison, the site specific proportional registration ratio (age adjusted) and the estimated standardised registration ratio

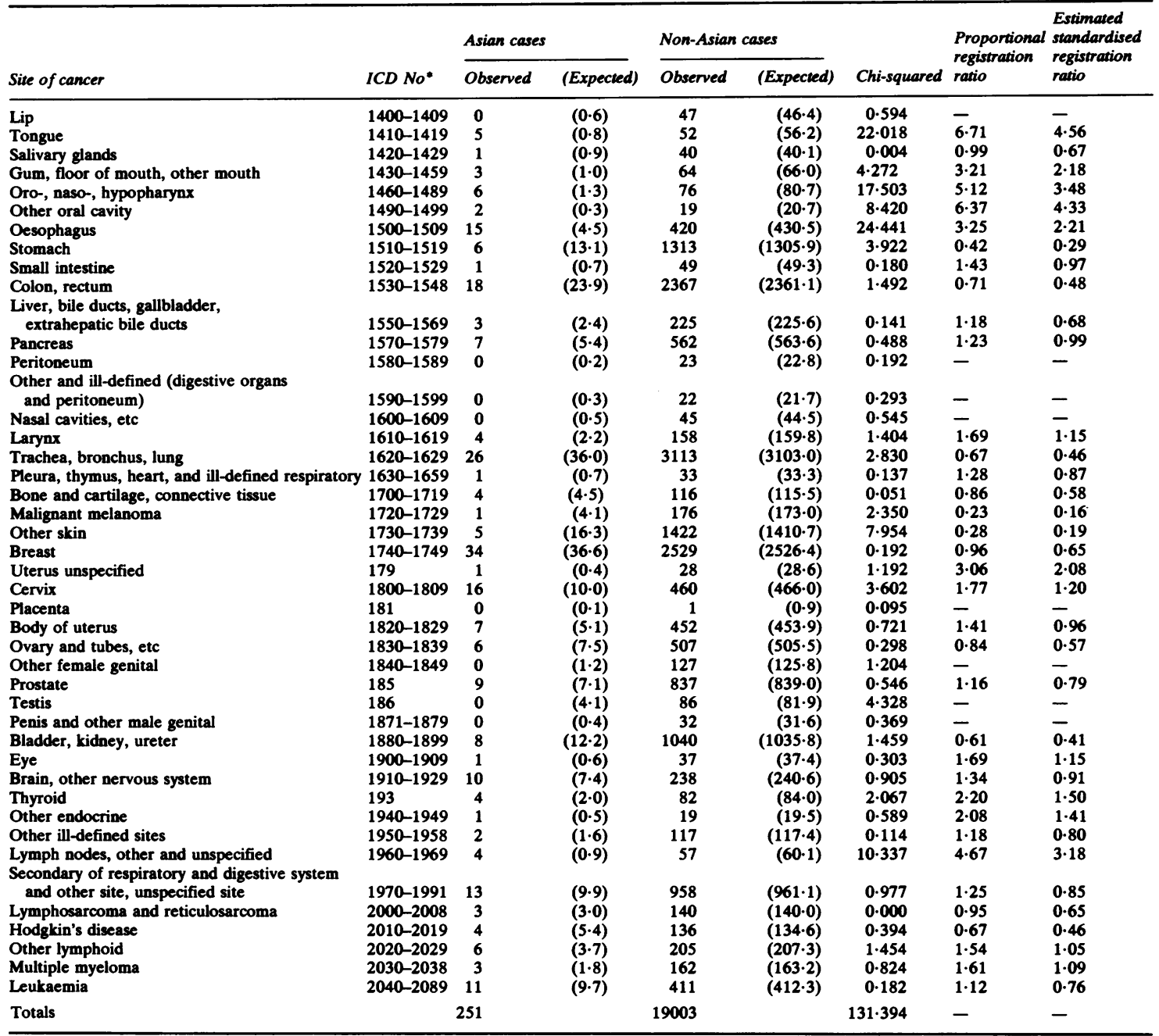

*International Classification of Diseases, 9th revision. Changes between 8th and 9th revision were reconciled during the analysis.

larger contributions to $\chi^{2}$ fell well outside the simulation envelopes, we feel justified in concluding that the distribution of cancer among sites was markedly different in Asians than in non-Asians, after taking account of the age distribution of cases.

Using this latter approach, the pronounced excess of Asian cases of oesophageal, tongue, pharyngeal, oral cavity, and other lymph node cancer was confirmed. Similarly, for skin cancer (other than melanoma) and testicular and stomach cancer, there appeared to be a significant deficit of Asian cases, although for the last two of these the difference was not so pronounced.

\section{Discussion}

It is only relatively recently that research workers have widened the study of disease problems among immigrant populations in Britain to encompass chronic diseases ${ }^{7}$ in addition to their more long standing concern with, for example, infectious and nutritional disorders. Even given this recent 


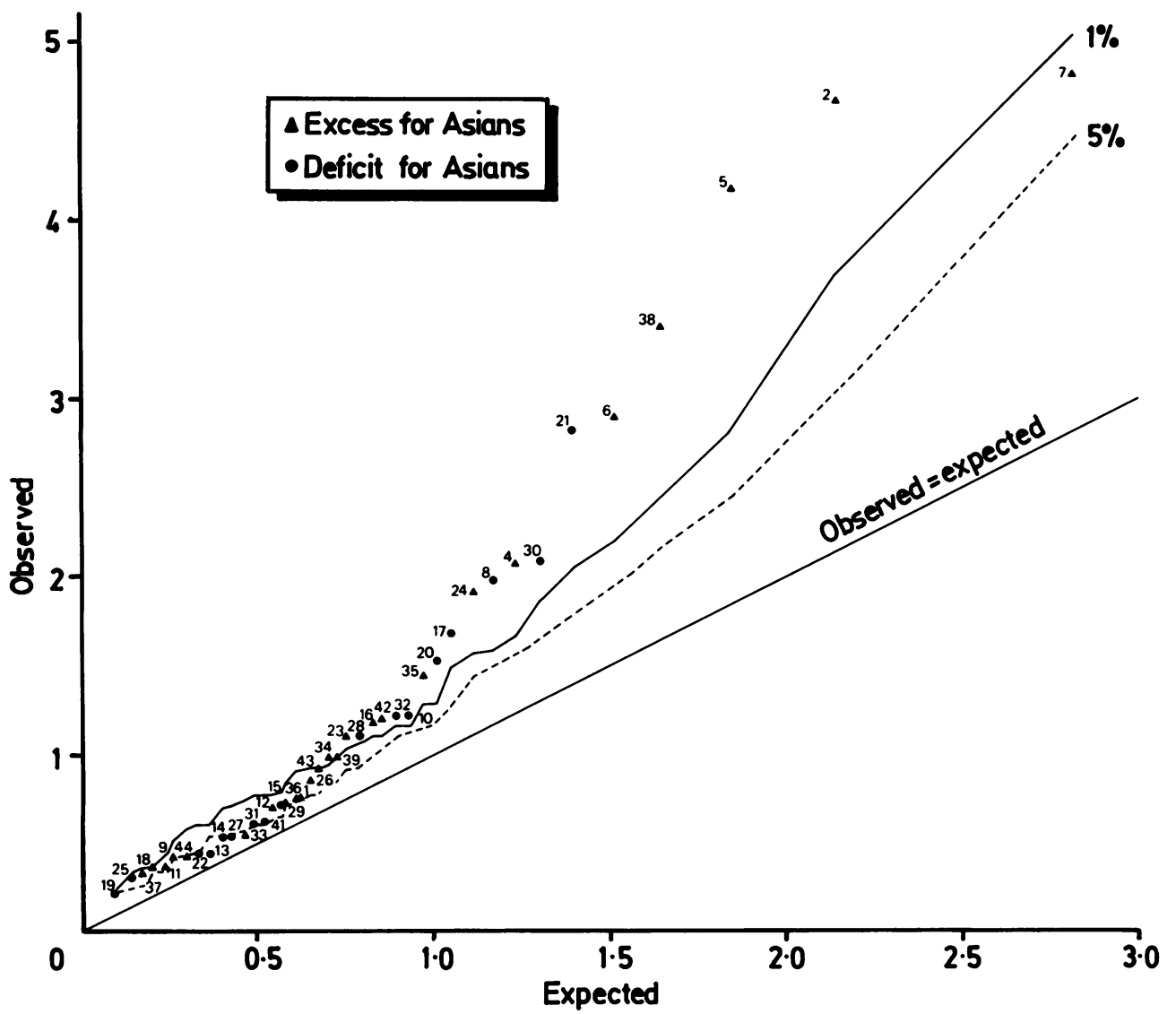

Contributions of each cancer site to the overall chi-squared test shown in the table, with simulation envelopes for $1 \%$ and $5 \%$ levels of significance log units.

Site of cancer*: 1 Lip, 2 tongue, 3 salivary glands, 4 gum, floor of mouth, other mouth, 5 oro-, naso-, hypopharynx, 6 other oral cavity, 7 oesophagus, 8 stomach, 9 small intestine, 10 colon, rectum, 11 liver, bile ducts, gallbladder, extrahepatic bile ducts, 12 pancreas, 13 peritoneum, 14 other and ill-defined (digestive organs, and peritoneum), 15 nasal cavities, etc, 16 larynx, 17 trachea, bronchus, lung, 18 pleura, thymus, heart, 19 bone and cartilage, connective tissue, 20 malignant melanoma, 21 other skin, 22 breast, 23 uterus unspecified, 24 cervix, 25 placenta, 26 body of uterus, 27 ovary and tubes, 28 other female genital, 29 prostate, 30 testis, 31 penis and other male genital, 32 bladder, kidney, ureter, 33 eye, 34 brain, other nervous system, 35 thyroid, 36 other endocrine, 37 other ill defined sites, 38 lymph nodes, other and unspecified, 39 secondary of respiratory and digestive system and other site, unspecified site, 40 lymphosarcoma and reticulosarcoma, 41 Hodgkin's disease, 42 other lymphoid, 43 multiple myeloma, and 44 leukaemia.

${ }^{*}$ Corresponds to same ICD groupings as in the table.

emphasis, there are few examples of studies based on disease occurrence in whole, rather than hospital, populations.

Cancer registration potentially affords such an opportunity in that it is a systematically collected source of data based on geographically defined populations. As is pointed out elsewhere, ${ }^{8}$ epidemiological studies that seek to explore reasons for variation in the incidence of cancer among different groups in the population may elucidate causes and perhaps enable new preventive initiatives to be taken. As indicated by the necessarily complex analysis used in this study, such comparisons between the immigrant and the indigenous population are not simple to make. Yet, when data were modified, and a methodology devised, to allow the disease experience of one particular ethnic group to be compared with the remainder of the population, potentially important differences were shown.

An alternative, spurious explanation of these differences would be that there is selective immigration into Britain of Asians who already have 
cancer at the time of entry. This is possible but seems somewhat implausible. Indeed, the apparent excess of cases of oral cancer in Asians compared with non-Asians, found here, is consistent with international comparisons which show that in India the annual age adjusted incidence rate of oral cancer in men was higher than for all men in the United Kingdom. ${ }^{9}$ Within countries, also, a higher occurrence of oral cancer has been reported in South Africa among Indians than in people of English origin $^{10}$ whereas, in the multiracial community of Singapore, Indian men and women have a higher incidence of these cancers than do Chinese or Malays. $^{{ }^{9}}$

It is well known that smoking ${ }^{112}$ and heavy alcohol consumption ${ }^{11}{ }^{13}$ are risk factors for the development of cancer of the oral cavity. Less convincing evidence exists to implicate two other postulated risk factors-dietary deficiencies and poor oral hygiene. Of particular relevance to discussion of the results of the present study is the habit of betel chewing. Betel is chewed in the form of the leaf and the nut. The betel nut (more correctly the areca nut, ${ }^{14}$ ) may be chewed alone but often it is wrapped within betel leaf. Other ingredients, particularly tobacco or lime, may be chewed with betel, often also by first wrapping them within the leaf.

In countries where betel is chewed its common accompaniment in the chew, tobacco, has been implicated in the aetiology of cancerous and precancerous lesions of the oral cavity. ${ }^{15}{ }^{16}$ It has further been suggested that elements in the betel chewing habit other than tobacco-that is, the areca nut, the betel leaf, or the lime additive-may be causally linked to oral cancer as well as to pharyngeal and oesophageal cancer. ${ }^{1017}$ An excess of oesophageal cancer was found among Asians in the present study.

Burton-Bradley is right to point to the lack of rigour in previous aetiological studies of this particular risk factor. ${ }^{14}$ Nevertheless, his implication of possible "ethnic and cultural bias" surely cannot be allowed to restrict studies in this field. Ethnic group is, in epidemiological terms, a variable to be examined in relation to disease patterns in the same way as others such as occupation, marital status, or age.

The Asian population of present day Britain is still relatively young in its age structure, consequently chronic diseases such as cancer are uncommon, as indicated by the relatively small number of observed Asian cases shown in the table. This will not always be so. As the population ages, there will be a need to be able to anticipate the main health problems of this community. Observational studies, such as this, can never prove cause and effect. They do, however, provide important pointers to areas, such as apparent associations found here, where formal epidemiological inquiry is indicated. Furthermore, they indicate a need to establish the prevalence within the ethnic minority population of traditional practices and behaviours that may be of public health importance.

Requests for reprints to Dr L J Donaldson.

\section{References}

${ }^{1}$ Donaldson LJ, Taylor JB. Patterns of Asian and non-Asian morbidity in hospitals. $\mathrm{Br}$ Med J 1983; 286: 948-51.

${ }^{2}$ Doll R, Peto R. The causes of cancer: quantitative estimates of the avoidable risks of cancer in the United States today. J Natl Cancer Inst 1981; 66: 1191-308.

${ }^{3}$ Donaldson LJ. In: The health problems of ethnic minorities. Report of the symposium held on the occasion of the 1983 Junior Members Forum. London: British Medical Association, 1983: 25-37.

'Sillitoe K. Ethnic origin: the search for a question. Population Trends 1978; 13: 25-30.

5 Jones PR. Research report: ethnic intermarriage in Britain. Ethnic and Racial Studies 1982; 5: 223-8.

- Anonymous. Sources of statistics on ethnic minorities [Editorial]. Population Trends 1982; 28: 1-8.

${ }^{7}$ Anonymous. Ethnic differences in common diseases. Postgrad Med J 1983; 59: 615-73.

${ }^{8}$ Office of Population Censuses and Surveys. Report of the advisory committee on cancer registration-cancer registration in the 1980s. London: HMSO, 1981.

${ }^{9}$ Waterhouse J, Muir C, Correa P, et al (eds). Cancer incidence in five continents. Vol III. Lyon: International Agency for Research on Cancer, 1976.

${ }^{10}$ Schonland M, Bradshaw E. Upper alimentary tract cancer in Natal Indians with special reference to the betel chewing habit. Br J Cancer 1969; 23: 670-82.

${ }^{11}$ Wynder EL, Bross IJ, Feldman R. A study of aetiological factors in cancer of the mouth. Cancer 1957; 10: 1300-23.

${ }^{12}$ Wynder EL, Stellman SD. Comparative epidemiology of tobacco-related cancers. Cancer Res 1977; 37: 4608-22.

${ }^{13}$ Rothman K, Kellar A. The effect of joint exposure to alcohol and tobacco on risk of cancer of the mouth and pharynx. J Chronic Dis 1972; 25: 711-6.

${ }^{14}$ Burton-Bradley BG. Is "betel chewing" carcinogenic? Lancet 1979; ii: 903.

${ }^{15}$ Ramanathan $\mathrm{K}$. Oral carcinoma in the first three decades of life. Med J Malaysia 1972; 27: 20-6.

${ }^{16}$ Wahi PN, Mital VP, Lahiri B, Luthra UK, Seth RK, Arora GD. Epidemiological study of precancerous lesions of the oral cavity: a preliminary report. Indian J Med Res 1970; 58: 1361-91.

${ }^{17}$ Stephen SJ, Uragoda CG. Some observations on oesophageal carcinoma in Ceylon, including its relationship to betel chewing. Br J Cancer 1970; 24: 11-5. 